specific ion channel inhibition, e.g. to allow selective antiarrhythmic drug development for the treatment of atrial fibrillation. Here, we sought to compare voltage-gated sodium currents $\left(\mathrm{I}_{\mathrm{NA}}\right)$ in atrial and ventricular cardiomyocytes in mice, as $\mathrm{Na}^{+}$channel subunits have been found to differ between atria and ventricles in rat and man.

Aim The aim of this study is to examine whether biophysical properties of $\mathrm{I}_{\mathrm{NA}}$ are altered in mouse atrial cardiomyocytes compared to left ventricular cardiomyocytes.

Methods $\mathrm{Na}+$ channel currents were measured using whole-cell voltage clamp in left atrial and left ventricular cardiomyocytes. Expression of Nav1.5 proteins and their regulatory ?-subunits was measured by western blotting in left atrial, right atrial and left ventricular tissue of wild-type 129/sv mice (15-20 weeks). Protein levels were normalised against calnexin.

Results Mean peak INA was significantly increased in left atrial myocytes compared to left ventricular $(\mathrm{LA}=-28.63 \pm 1.856 \mathrm{pA} /$ $\mathrm{pF} ; \mathrm{n}=15 / 4$ cells/mice; $\mathrm{LV}=-19.83 \pm 4.186 \mathrm{pA} / \mathrm{pF} ; \mathrm{n}=5 / 2$ cells/ mice; $* \mathrm{p}<0.05$ ) and V50 for INA inactivation was significantly more negative in left atrial compared to left ventricular myocytes $(\mathrm{LA}=-92.4 \pm 1.877 \mathrm{mV} ; \quad \mathrm{n}=16 / 4 \quad$ cells $/$ mice; $\quad \mathrm{LV}=-81.77$ $\pm 2.413 \mathrm{mV} ; \mathrm{n}=5 / 2$ cells/mice; $* \mathrm{p}<0.01)$. No difference in Nav1.5 expression was detected between chambers, however, expression of $\tilde{\mathrm{A}} \square \hat{\mathrm{A}}^{2} 2$ and $\tilde{\mathrm{A}} \square \hat{\mathrm{A}}^{2} 4$ subunits was significantly reduced in atrial tissue compared to left ventricular ( $\mathrm{LA}=0.189$ $\pm 0.02014 ; \mathrm{RA}=0.3023 \pm 0.0333 ; \mathrm{LV}=0.736 \pm 0.0718 ;$ * $\mathrm{p}<0.01$; $\mathrm{n}=4) \quad$ and $\quad(\mathrm{LA}=0.00145 \pm 0.00033 ; \mathrm{RA}=0.00204 \pm 0.00102$; $\mathrm{LV}=0.0214 \pm 0.000613 ; * \mathrm{p}<0.01 ; \mathrm{n}=4)$ respectively.

Conclusion Mouse atrial cardiomyocytes display increased INA compared to cardiomyocytes isolated from the ventricles. Alterations in biophysical properties of INA in mouse atrial myocytes may be attributable to reduced expression of the Nav1.5 $\tilde{\mathrm{A}} \square \hat{\mathrm{A}}^{2} 2$ and Nav1.5 $\tilde{\mathrm{A}} \square \hat{\mathrm{A}}^{2} 4$ subunits. Considering the interaction between Nav1.5 and its $\tilde{\mathrm{A}} \square \hat{\mathrm{A}}^{2}$ subunits may provide novel targets for antiarrhythmic drug therapy.

\section{AUGMENTATION OF CREATINE KINASE IN VITRO PROTECTS AGAINST SIMULATED ISCHAEMIA REPERFUSION INJURY}

Sevasti Zervou*, Hannah J Whittington, Philip J Ostrowski, Fang Cao, Jack Tyler, Hannah A Lake, Stefan Neubauer, Craig A Lygate. University of Oxford

\subsection{6/heartjnl-2017-311726.220}

Creatine kinase (CK) catalyses the interchange of high energy phosphates to buffer ATP levels and maintains cellular energy homeostasis. The heart expresses three isoforms: sarcomeric mitochondrial CK (CKMT2), and the cytoplasmic CKM and $\mathrm{CKB}$ isoforms which form homo (MM/BB)- and hetero (MB)dimers. Impaired $\mathrm{CK}$ activity is associated with heart failure and increases susceptibility to ischaemia/reperfusion injury.

We hypothesised that augmentation of $\mathrm{CK}$ isoenzymes in vitro would improve cell viability following exposure to hypoxia/reoxygenation. For this purpose we created CK overexpression systems by cloning the open reading frame of the different CK isoform sequences into pcDNA3.1 expression vector and stably selected and characterised overexpressing HEK293 cell lines.

The generated cell lines displayed increased CK activity in addition to individual CK isoenzyme activities. CKMT2, CKM and CKB cells had elevated total CK activity $(\mathrm{p}<0.001$; $\mathrm{p}<0.001 ; \mathrm{p}<0.01$ One-way ANOVA, Dunnetts post-test vs
HEK293). Furthermore immunocytochemistry showed that CKMT2 co-localises with mitochondrial marker COXIV in the intermembrane space following transient transfection in HL1 atrial cell line.

Both stable and untransfected HEK293 cells were exposed to simulated ischaemia/reperfusion by incubating at $1 \% \mathrm{O}_{2}$ for 18 hour, followed by re-oxygenation at 95\% $\mathrm{O}_{2}$ for 2 hour. The positive control rapamycin was supplemented into the cell media 4 hours prior to hypoxia. Viability analysis by propidium iodide detection using a CyAN flow cytometer at $488 \mathrm{~nm}$, showed increased cell survival by 33\% in CKMT2, $47 \%$ in CKM and $58 \%$ in CKB cells when compared to untransfected HEK293 controls (in all cases p $<0.05$, One-Way ANOVA Dunnetts post-test vs HEK293).

To determine whether protection was due to changes in antioxidant capacity, cells were loaded with the reactive oxygen species indicator dye, $\mathrm{DCFH}_{2}-\mathrm{DA}$, and exposed to $\mathrm{H}_{2} \mathrm{O}_{2}$ induced oxidative stress. Overexpression of CK isoenzymes failed to attenuate fluorescence from oxidised dye in contrast to the known antioxidant, Trolox. Transient expression of CK constructs in the HL1 cell line was used to test the effects of anthracycline exposure on cell viability (48 hour doxorubicin). Pre-treatment with Trolox increased cell survival by $12.4 \%$ $(79.4 \% \pm 2.0$ vs. $67 \% \pm 1.5$ in empty-vector control cells; $\mathrm{p}<0.01$ ) whereas overexpression of $\mathrm{CK}$ isoenzymes did not alter cell death rates.

In conclusion, overexpression of any one (of three) cardiac creatine kinase isoenzymes protects against ischemia/reperfusion in vitro. This most likely reflects enhanced energy reserve due to elevated CK activity, since response to oxidative challenge was unaltered. Further mechanistic studies and in vivo confirmation of these findings are merited.

\section{INVESTIGATING THE ROLE OF AEROBIC GLYCOLYSIS IN ARTERIAL CALCIFICATION}

Nabil Rashdan, Vicky MacRae*. University of Edinburgh

\subsection{6/heartjnl-2017-311726.221}

Objective The process of arterial calcification shares many similarities to skeletal mineralisation, and involves the deposition of hydroxyapatite in the arteries. However, the cellular mechanisms responsible have yet to be fully elucidated. Accumulating evidence suggests that aerobic glycolysis (the Warburg effect), plays a critical role in meeting the demand for energy and biosynthetic precursors during proliferation and differentiation in numerous cell types. Therefore we addressed the hypothesis that vascular smooth muscle cell (VSMC) calcification requires aerobic glycolysis to produce energy and the necessary biosynthetic precursors.

Methods Calcification of murine aortic VSMCs was induced by $3 \mathrm{mM}$ Pi for 7 days. Calcium deposition was determined using alizarin red staining and a modified o-cresolphthalein method. VSMCs were cultured with the fluorescent glucose analogue 2-(N-(7-Nitrobenz-2-oxa-1,3-diazol-4-yl)Amino)-2Deoxyglucose (2-NBDG) to determine changes in glucose uptake. Gene expression was analysed by qRT-PCR.

Results Calcium deposition was significantly increased in VSMCs cultured in $3 \mathrm{mM}$ Pi versus control conditions (124\%, $\mathrm{p}<0.001)$. Calcified VSMCs also showed increased mRNA expression of Runx2, Phospho1, Ocn and PiT-1 $(\mathrm{p}<0.001)$, recognised osteogenic markers of arterial calcification. 